\title{
L-fucose ameliorates the carcinogenic properties of Fusobacterium nucleatum in colorectal cancer
}

\author{
CAIHAN DUAN $^{1}$, XUELIAN TANG $^{1}$, WEIJUN WANG ${ }^{1}$, WEI QIAN $^{1}$, XIAOCHAO FU $^{2}$, \\ XIAOHUA DENG ${ }^{2}$, CHAOQUN HAN ${ }^{1}$ and XIAOHUA HOU ${ }^{1}$
}

${ }^{1}$ Division of Gastroenterology, Union Hospital, Tongji Medical College, Huazhong University of Science and
Technology; ${ }^{2}$ Hubei Center of Industrial Culture Collection and Research, Wuhan, Hubei 430020, P.R. China

Received May 6, 2020; Accepted November 13, 2020

DOI: 10.3892/ol.2020.12404

\begin{abstract}
Fusobacterium nucleatum $(F n)$ is considered a promoting factor in colorectal cancer (CRC); however, only a few studies have investigated therapies against $F n$. L-fucose is a natural monosaccharide that has prebiotic potential. The present study aimed to investigate the effect of L-fucose on the carcinogenic properties of Fn. The HCT116 and SW480 colon cancer cell lines were treated with $F n$ and $F n+\mathrm{L}$-fucose $(F n f)$, respectively. The Cell Counting Kit-8 (CCK-8), colony formation, Transwell migration and invasion and wound healing assays were performed to assess the proliferative, migratory and invasive abilities of the cells, respectively. Western blot was performed to detect the protein levels of jak/stat 3 pathway components and EMT. The results of the CCK-8, colony formation, Transwell and wound healing assays demonstrated that treatment with $F n$ significantly enhanced the proliferative, migratory and invasive abilities of HCT116 and SW480 colon cancer cells. Notably, these effects were significantly reversed following addition of L-fucose. Furthermore, L-fucose inhibited the carcinogenic properties of $F n$ to activate the stat 3 pathway and epithelial-to-mesenchymal transition. Taken together, the results of the present study suggest that L-fucose ameliorates the carcinogenic properties of $F n$ in vitro, and thus may serve as a novel therapeutic target for flora-related colon cancer.
\end{abstract}

\section{Introduction}

Colorectal cancer (CRC) is the fourth most common cancer worldwide (6.1\% of total cases in 2018) (1). Despite

Correspondence to: Professor Xiaohua Hou or Dr Chaoqun Han, Division of Gastroenterology, Union Hospital, Tongji Medical College, Huazhong University of Science and Technology, 1277 Jiefang Avenue, Wuhan, Hubei 430020, P.R. China

E-mail: hcq1987912@hotmail.com

E-mail: houxh@hust.edu.cn

Key words: L-fucose, colorectal cancer, Fusobacterium nucleatum, Stat3, epithelial-to-mesenchymal transition advancements in its diagnosis and treatment strategies, CRC remains the leading cause of cancer-associated mortality worldwide $(9.2 \%$ of the total cancer-associated deaths in 2018) (1). Thus, better understanding the molecular mechanism of CRC progression and identifying novel effective therapeutic strategies remains essential.

Gut microbiome is closely associated with the development of CRC (2). Recently, the Gram-negative anaerobic bacteria, Fusobacterium nucleatum (Fn), has gained great interest. Previous studies have demonstrated that $F n$ is enriched in CRC tissues compared with adjacent normal tissues, and associated with poor prognosis $(3,4)$. In addition, $F n$ promotes the development of CRC by activating autophagy of cancer cells, protecting tumors from immune attack and creating an inflammatory microenvironment $(5,6)$. Although it has been reported that using berberine and targeting $F n$ Fap2 may decrease $F n$ potentiation of CRC $(7,8)$, methods for suppressing the carcinogenic properties of $F n$ remain largely unknown.

L-Fucose (Fucose), a natural monosaccharide present in foods or bodies, plays an important role in sustaining the gut homeostasis (9). Our previous studies demonstrated that fucose alleviates dextran sulfate sodium-induced acute and chronic colitis by regulating immune responses and affecting the intestinal microenvironment $(10,11)$. Notably, fucose has been reported to affect microbial metabolic pathways, and decrease pathogen virulence (12). However, the role of fucose in CRC remains unknown.

Thus, the present study aimed to investigate the effects of fucose on the functional regulations of $F n$ and determine its underlying molecular mechanism in CRC. Taken together, the results of the present study suggest that fucose may ameliorate the carcinogenic properties of $F n$.

\section{Materials and methods}

Cell lines, bacterial strain culture and groups. The HCT116 and SW480 human colon cancer cell lines were purchased from the American Type Culture Collection and maintained in high glucose DMEM supplemented with $10 \%$ fetal bovine serum (FBS) and $100 \mathrm{U} / \mathrm{ml}$ streptomycin/penicillin (all purchased from Gibco; Thermo Fisher Scientific, Inc.), at $37^{\circ} \mathrm{C}$ in $5 \% \mathrm{CO}_{2}$.

$F n$ (25586) was purchased from the American Type Culture Collection and cultured at Wuhan Research Institute of First 
Light Industry (Wuhan, China). The methods of bacterial pellets and conditioned medium were performed, as previously described (13). The following groups were classified: Control group, $F n$ group (supernatant of $F n$ was added to the cells) and $0.5 \%$ fucose+Fn $(F n f)$ group. Fucose (Sigma-Aldrich; Merck $\mathrm{KGaA}$ ) was added when $F n$ was cultured and the supernatant was subsequently added to the cells. In total, $100 \mathrm{nM}$ S3I-201 (Selleck Chemicals), an inhibitor of stat3, was incubated for $1 \mathrm{~h}$ prior to bacteria treatment to access the role of stat 3 pathway.

Cell Counting Kit-8 (CCK-8) assay. The CCK-8 assay was performed to assess cell proliferation. HCT116 and SW480 cells were seeded into a 96-well plate at a density of $1 \times 10^{4}$ with $100 \mu \mathrm{l}$ conditioned medium and allowed to adhere overnight. Following treatment with $F n$ or $F n f$ for $12 \mathrm{~h}$ at $37^{\circ} \mathrm{C}$, the culture medium was removed and $10 \mu \mathrm{l}$ CCK-8 solution (Beyotime Institute of Biotechnology) along with $90 \mu \mathrm{l}$ medium was added to each well. Following incubation for $1 \mathrm{~h}$ at $37^{\circ} \mathrm{C}$, cell proliferation was analyzed at a wavelength of $450 \mathrm{~nm}$, using a microplate reader (Biotek Instruments, Inc.).

Colony formation assay. The colon cancer cells were stimulated with $F n$ or $F n f$ for $12 \mathrm{~h}$ at $37^{\circ} \mathrm{C}$ and subsequently seeded into 6-well plates at a density of 500 cells/well. Cells were cultured for 14 days at $37^{\circ} \mathrm{C}$ in $5 \% \mathrm{CO}_{2}$. Following incubation, cells were washed with PBS for three times, fixed with $4 \%$ paraformaldehyde for $30 \mathrm{~min}$ and stained with $0.5 \%$ crystal violet for $15 \mathrm{~min}$ both at room temperature. Cell colonies were counted using ImageJ1 software (National Institutes of Health).

Wound healing assay. Cells from each group were respectively seeded into 6-well plates at a density of $5 \times 10^{5}$ with serum-free DMEM medium and incubated overnight at $37^{\circ} \mathrm{C}$. Cell monolayers were scratched using a sterile pipette tip. Cells were washed with PBS three times to remove the cellular debris, and cell migration was subsequently analyzed after $24 \mathrm{~h}$, using ImageJ1 software (National Institutes of Health).

Transwell migration and invasion assays. For the cell migration assay, cells from each group were resuspended with serum-free DMEM medium and plated in the upper chambers of 24-well Transwell plates at a density of $1 \times 10^{5}$. DMEM medium supplemented with $10 \%$ FBS was plated in the lower chambers. Following incubation for $24 \mathrm{~h}$ at $37^{\circ} \mathrm{C}$, the migratory cells were fixed with $4 \%$ paraformaldehyde for $30 \mathrm{~min}$ and stained with $0.5 \%$ crystal violet for $15 \mathrm{~min}$ both at room temperature. Cells were observed under a light microscope (magnification x200; Olympus Corporation). For the cell invasion assay, Transwell membranes were precoated with Matrigel at $37^{\circ} \mathrm{C}$ for $4 \mathrm{~h}$.

Western blotting. Total protein was extracted from cells or tissues using RIPA lysis buffer (Beyotime Institute of Biotechnology) supplemented with phenylmethyl sulfonyl fluoride protease inhibitor (Beyotime Institute of Biotechnology) and phosphatase inhibitor (Beyotime Institute of Biotechnology). Total protein was quantified using the BCA assay (Thermo Fisher Scientific, Inc.) and equal amounts of protein (40 $\mu \mathrm{g} / \mathrm{lane})$ were separated by $10 \%$ SDS-PAGE. The separated proteins were subsequently transferred onto PVDF membranes (EMD Millipore) and blocked with 5\%
BSA at room temperature for $1 \mathrm{~h}$. The membranes were incubated with primary antibodies (all 1:1,000 dilution) against: GAPDH (ABclonal Biotech Co., Ltd.; cat. no. AC001), ACTB (ABcloanl Biotech Co., Ltd.; cat. no. AC006), phospho-stat3 (Tyr705; Cell Signaling Technology, Inc.; cat. no. 9145S), stat3 (ABclonal Biotech Co., Ltd.; cat. no. A11216), phosphor-jak2 (Tyr1007/1008; ABclonal Biotech Co., Ltd.; cat. no. AP0531), jak2 (ABclonal Biotech Co., Ltd.; cat. no. A7694), $\beta$-catenin (Cell Signaling Technology, Inc.; cat. no. 8480S), E-cadherin (Cell Signaling Technology, Inc.; cat. no. 3195S), N-cadherin (GeneTex, Inc.; cat. no. GTX127345) and Vimentin (Cell Signaling Technology, Inc.; cat. no. 5741S) overnight at $4^{\circ} \mathrm{C}$. Following the primary incubation, membranes were incubated with HRP-labelled secondary antibodies (AntGene; cat. no. ANT020; 1:2,000) at room temperature for $1 \mathrm{~h}$. Protein bands were visualized using enhanced chemiluminescent reagents (Beyotime Institute of Biotechnology).

Statistical analysis. Statistical analysis was performed using SPSS 25.0 software (IBM Corp.) and GraphPad Prism 7.0 software (GraphPad Software, Inc.). All experiments were performed in triplicate and data are presented as the mean \pm standard error of the mean. One-way analysis of variance and Tukey's post hoc test were used to compare differences between multiple groups. $\mathrm{P}<0.05$ was considered to indicate a statistically significant difference.

\section{Results}

Fucose ameliorates the pro-proliferation characteristic of Fn on colon cancer cells. To investigate whether there were differences between the impacts that $F n$ and $F n f$ exerted on colon cancer cells, the CCK-8 assay was performed on HCT116 and SW480 cells following treatment for $12 \mathrm{~h}$. The results demonstrated that cells treated with $F n$ proliferated faster compared with the control group (HCT116 control, $0.42 \pm 0.01$; $F n, 0.60 \pm 0.02, \mathrm{P}<0.0001$; SW480 control, $0.36 \pm 0.02 ; F n$, $0.42 \pm 0.01, \mathrm{P}=0.005$; Fig. $1 \mathrm{~A})$. However, following treatment with fucose, $F n$ exerted a weaker pro-proliferative ability (HCT116 Fn, 0.60 \pm 0.02 ; Fnf, 0.54 $\pm 0.01, \mathrm{P}=0.014 ;$ SW480 Fn, $0.42 \pm 0.01 ;$ Fnf, $0.38 \pm 0.01, \mathrm{P}=0.009$; Fig. 1A). The colony formation assay was subsequently performed to assess the long-term effect on cell proliferation. The results demonstrated that the number of HCT116 colonies treated with $F n$ increased (control, 52.0 $\pm 7.0 ; F n, 125.3 \pm 11.3 ; \mathrm{P}=0.005$ ), while less colonies were observed in the $F n f$ group ( $F n, 125.3 \pm 11.3$; Fnf, 81.0 $\pm 9.9, \mathrm{P}=0.042$; Fig. 1B and C). Similar results were observed in SW480 cells (control, 96 $\pm 10 ; F n, 170 \pm 5 ; F n f$, 131.5 $\pm 6.5 ; \mathrm{P}=0.022$ and $\mathrm{P}=0.043$; Fig. $1 \mathrm{~B}$ and $\mathrm{C}$ ). Collectively, these results suggest that L-fucose may ameliorate the pre-proliferative ability of $F n$ on colon cancer cells.

Fucose ameliorates the pro-migratory ability of Fn on colon cancer cells. The migratory ability of colon cancer cells is a functional characteristic to assess aggressiveness (14). The Transwell migration assay was performed to assess the effect of $F n$ and $F n f$ on the migratory ability of HCT116 and SW480 cells. The results demonstrated that treatment with $F n$ significantly enhanced the migratory ability of HCT116 and SW480 cells (HCT116 control, 119.30 $\pm 3.84 ; F n, 148.30 \pm 4.41, \mathrm{P}=0.008$; 
A

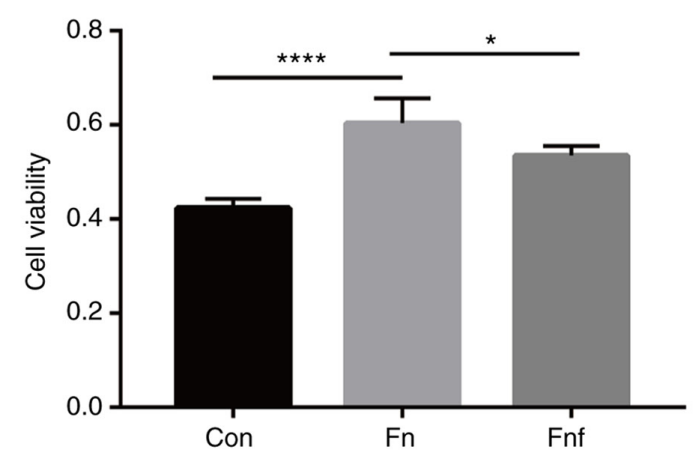

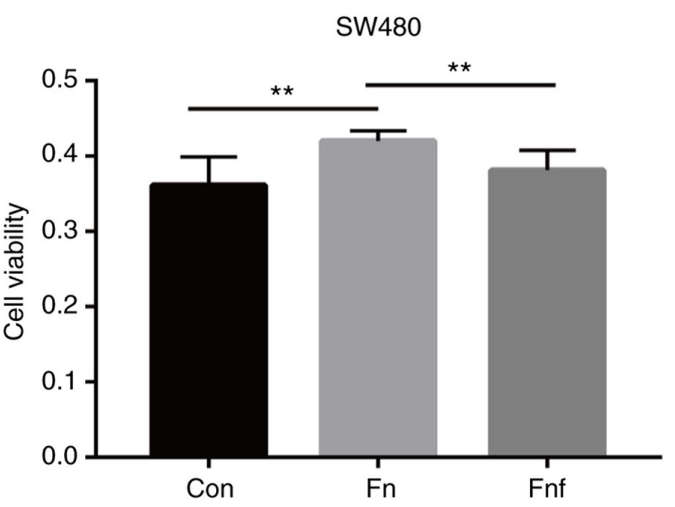

$\mathrm{Fn}$
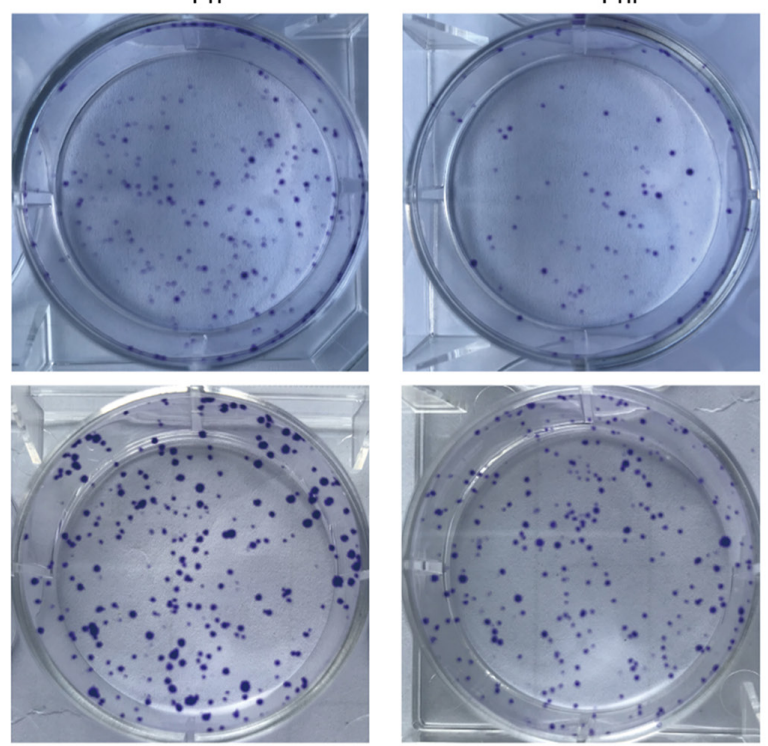

C
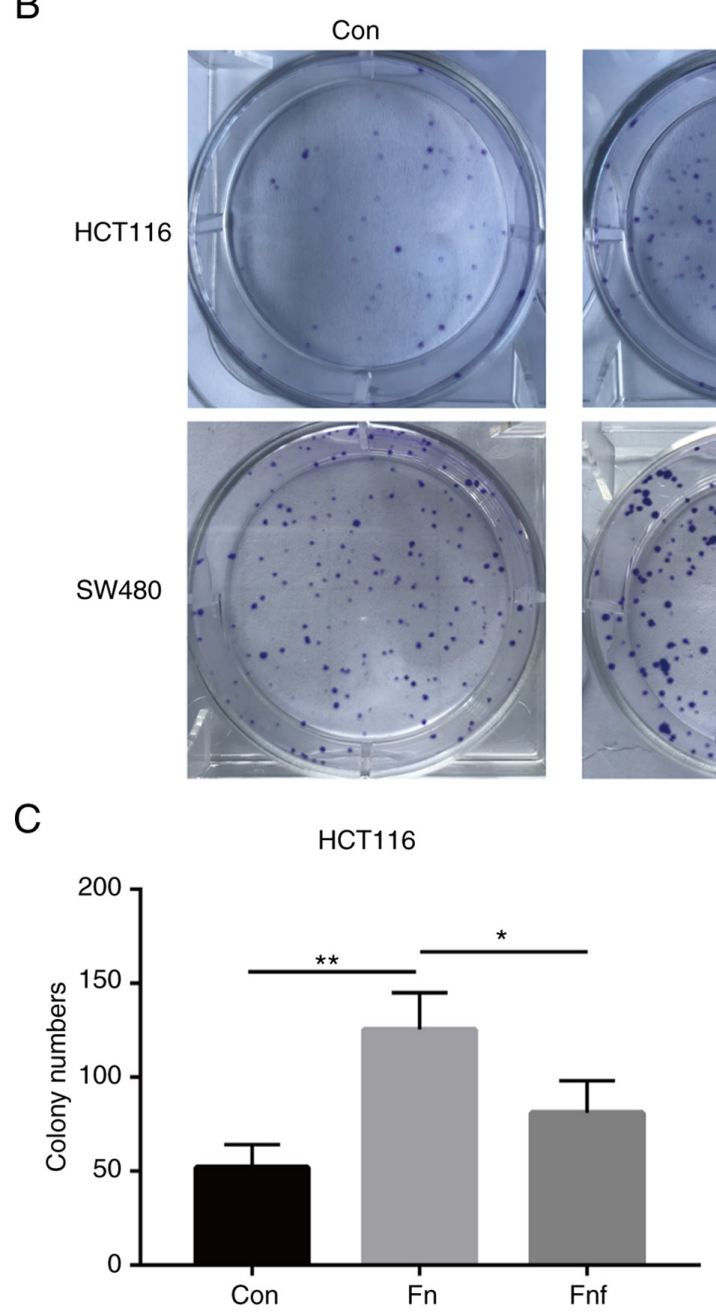

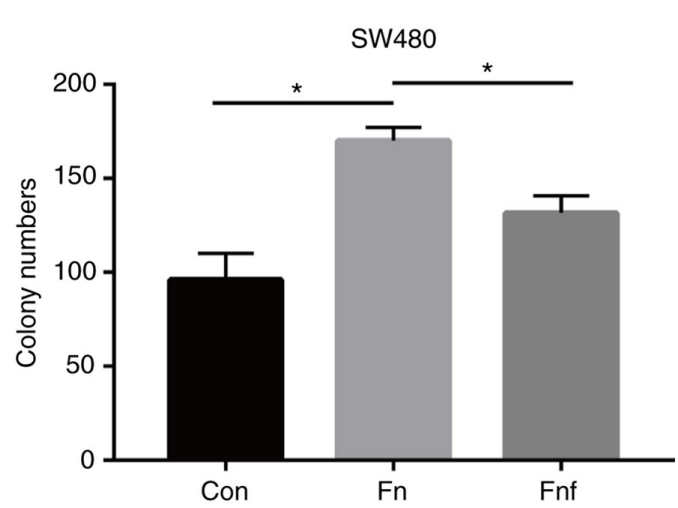

Figure 1. Fucose ameliorates the pro-proliferative ability of $F n$ on colon cancer cells. (A) The Cell Counting Kit- 8 and (B) colony formation assays were performed to assess proliferation of HCT116 and SW480 cells following different treatments. (C) Cell colonies were calculated using ImageJ1. Data are presented as the mean \pm standard error of the mean of at least three repeated experiments. ${ }^{*} \mathrm{P}<0.05,{ }^{* *} \mathrm{P}<0.01,{ }^{* * * *} \mathrm{P}<0.0001$. Fn, Fusobacterium nucleatum; Fnf, Fn+L-fucose; Con, control.

SW480 control, $123.00 \pm 4.93 ; F n, 216.50 \pm 3.30 ; \mathrm{P}<0.0001$ Fig. 2A), while the migratory ability decreased in cells treated with Fnf (HCT116 Fn, 148.30 $\pm 4.41 ; F n f, 128.80 \pm 2.29, \mathrm{P}=0.008$; SW480 Fn, 1216.50 $\pm 3.30 ; F n f, 157.70 \pm 2.96, \mathrm{P}=0.002$; Fig. 2A).

The results of the wound healing assay demonstrated that treatment with $F n$ increased the migratory rate in HCT116 cells compared with both the control cells and $F n f$ treated cells (control, 0.152 $\pm 0.006 ;$ Fn, 0.313 $\pm 0.020 ;$ Fnf, 0.221 \pm 0.011 ; $\mathrm{P}=0.002$ and $\mathrm{P}=0.018$; Fig. $2 \mathrm{~B}$ ). Similar trends were observed in SW480 cells (control, 0.190 $\pm 0.011 ; F n, 0.410 \pm 0.016$; Fnf, 0.259 $\pm 0.029 ; \mathrm{P}<0.0001$ and $\mathrm{P}=0.004$; Fig. 2C). Taken together, these results suggest that L-fucose may inhibit the pro-migratory ability of $F n$ on colon cancer cells.

Fucose ameliorates the pro-invasive ability of Fn on colon cancer cells. The Transwell invasive assay was performed to assess the effect of $F n$ and $F n f$ on the invasive ability of HCT116 and SW480 cells. The number of HCT116 and 
A
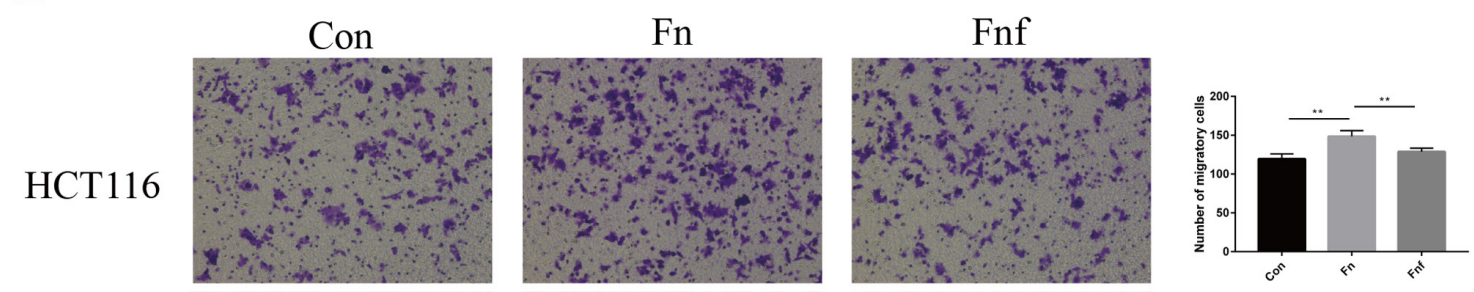

SW480
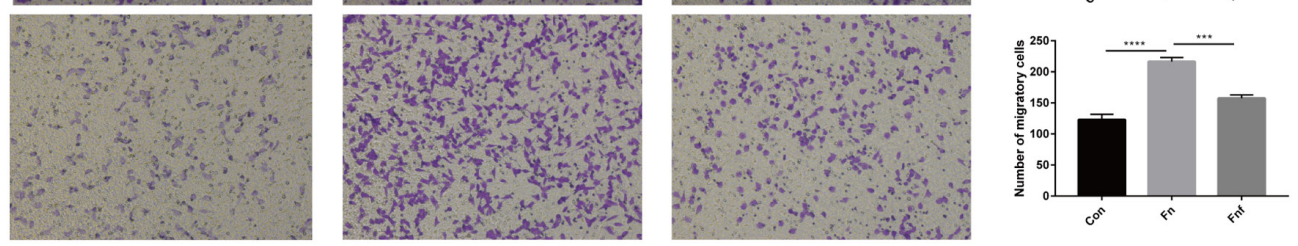

B

$0 \mathrm{~h}$

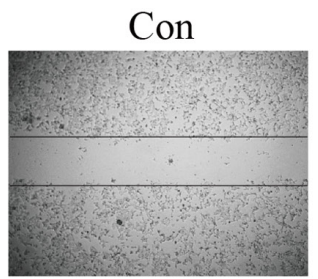

Fn
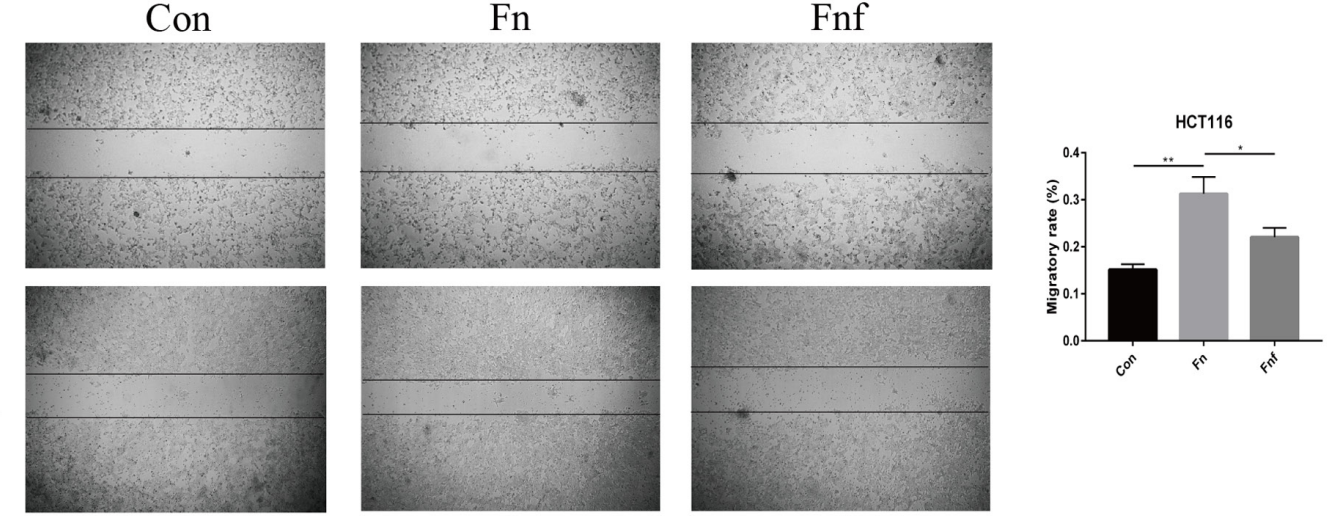

C

Con

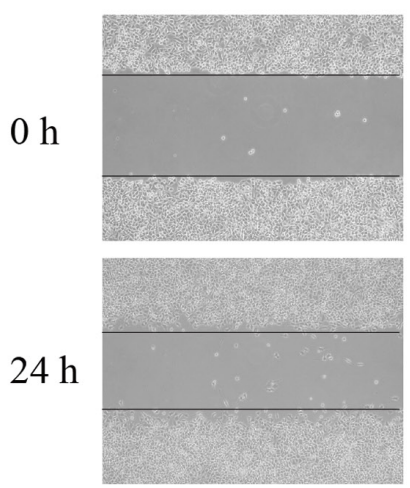

Fn
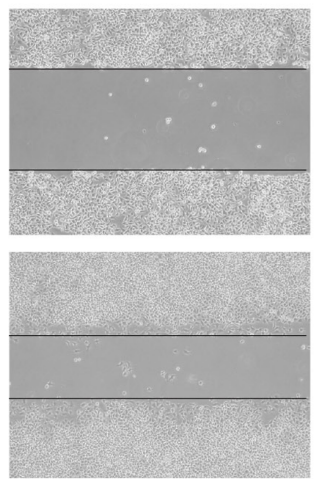

Fnf
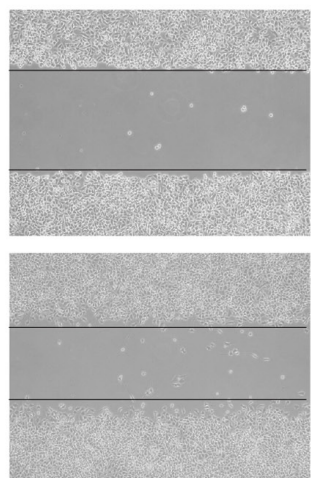

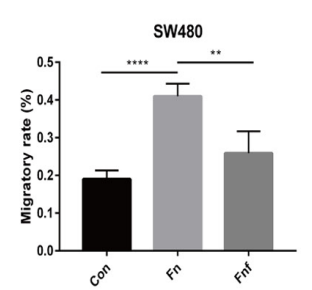

Figure 2. Fucose ameliorates the pro-migratory ability of $F n$ on colon cancer cells. (A) The Transwell migration assay was performed to assess the migratory ability of HCT116 and SW480 cells following different treatments. The wound healing assay was performed to assess the migratory rate of (B) HCT116 and (C) SW480 cells following different treatments. Data are presented as the mean \pm standard error of the mean of at least three repeated experiments. "P<0.05, ${ }^{* * *} \mathrm{P}<0.01,{ }^{* * * *} \mathrm{P}<0.001,{ }^{* * * * *} \mathrm{P}<0.0001$. Fn, Fusobacterium nucleatum; Fnf, Fn+L-fucose; Con, control.

SW480 cells that passed through the membrane was significantly higher in the $F n$ group compared with the control group (HCT116 control, 111.50 $\pm 15.16 ; F n, 234.00 \pm 14.70$, $\mathrm{P}=0.001$; Fig. 3A and B; SW480 control, 500.00 $\pm 44.77 ; F n$, $857.80 \pm 50.50, \mathrm{P}=0.002$; Fig. $3 \mathrm{C}$ and D). As expected, the number of cells in the $F n f$ group significantly decreased compared with the $F n$ group (HCT116 Fn, 234.00 \pm 14.70 ; Fnf, 137.30 $\pm 27.53, \mathrm{P}=0.021$; Fig. $3 \mathrm{~A}$ and $\mathrm{B}$; SW480 Fn,

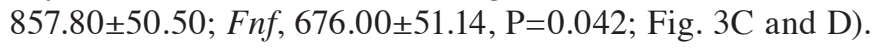
Collectively, these results suggest that L-fucose may ameliorate the pro-invasive ability of $F n$ on colon cancer cells.
Fucose ameliorates the carcinogenic property of Fn to activate stat 3 and EMT. The effect of fucose on Fn potentiation on activation of the pathway that promotes CRC progression was assessed in the present study. Stat 3 plays a crucial role in tumorigenesis, and progression and invasion of cancer cells (15). Thus, the expression and phosphorylation of stat 3 were detected via western blot analysis. As presented in Fig. 4, the protein levels of p-stat 3 and p-jak2 significantly increased following treatment with Fn (for p-stat3/stat3, HCT116 control, $1.000 \pm 0.020 ; F n, 1.851 \pm 0.063 ; \mathrm{P}=0.002$; SW480 control, $1.000 \pm 0.006 ; F n, 1.759 \pm 0.075 ; \mathrm{P}=0.004$. For p-jak2/jak2, HCT116 control, 1.000 $\pm 0.127 ; F n, 2.272 \pm 0.098$; 
A
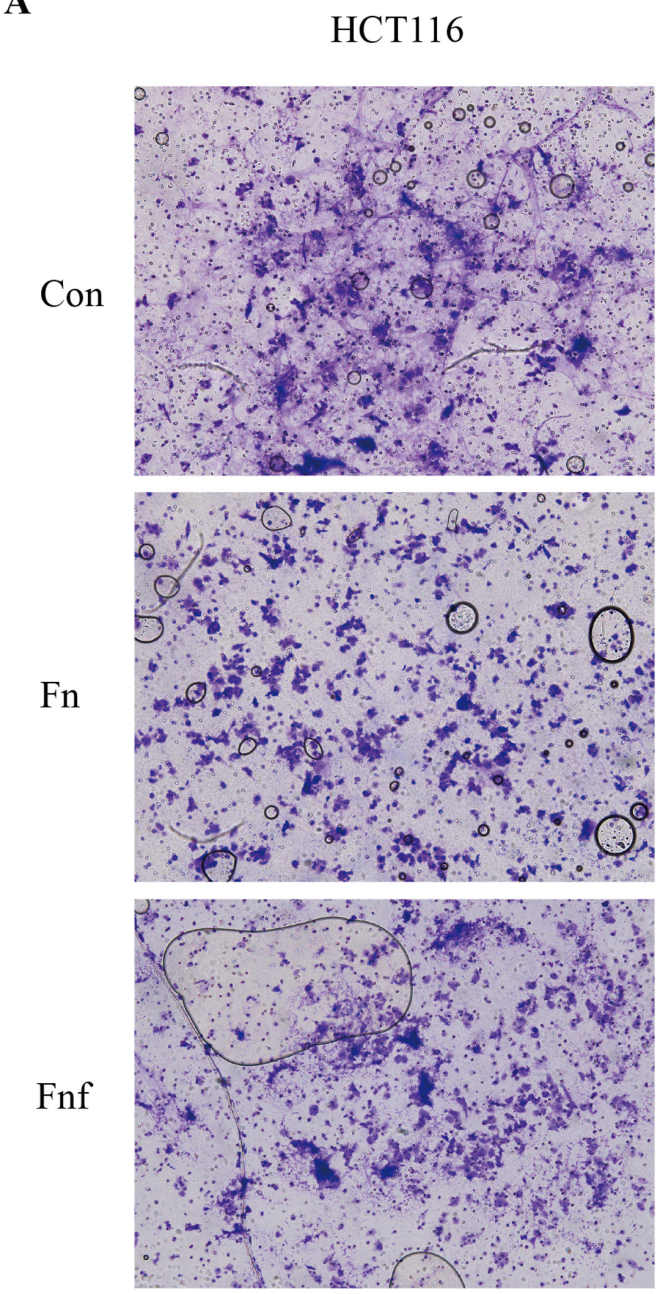

B

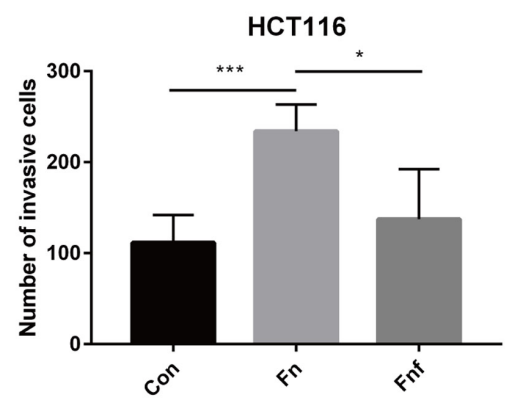

C
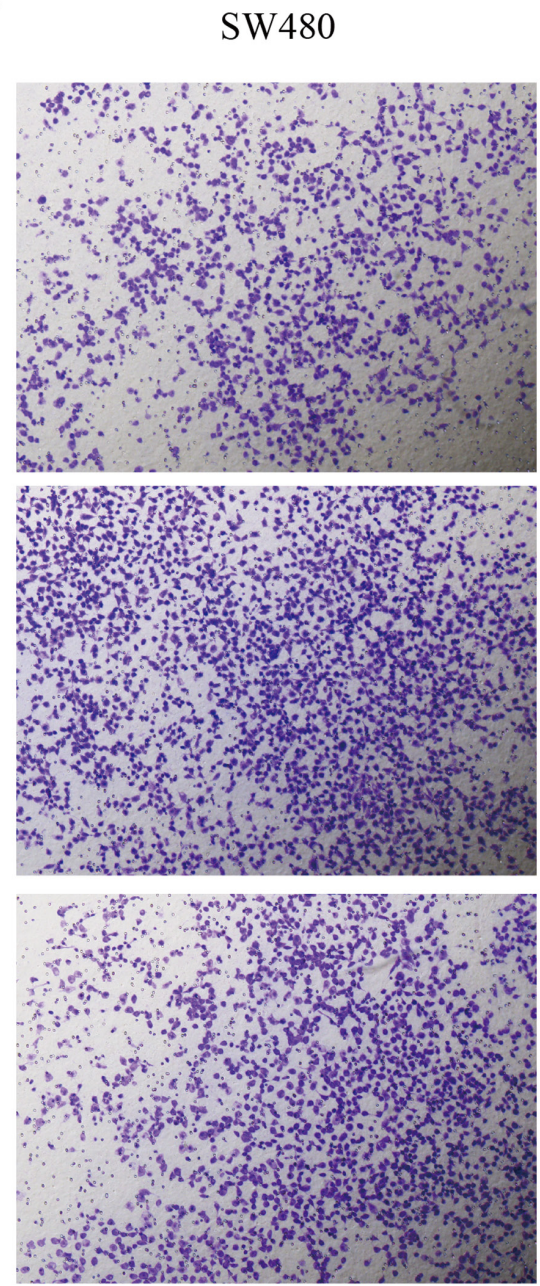

D

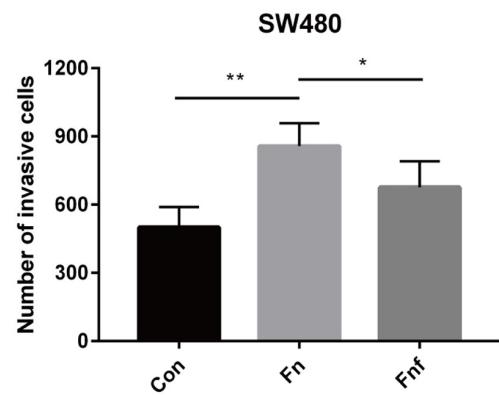

Figure 3. Fucose ameliorates the pro-invasive ability of $F n$ on colon cancer cells. (A) The Transwell invasion assay was performed on HCT116 cells following different treatments and (B) the number of invasive HCT116 cells was calculated. (C) The Transwell invasion assay was performed on SW480 cells following different treatments and (D) the number of invasive SW480 cells was calculated. Data are presented as the mean \pm standard error of the mean of at least three repeated experiments. ${ }^{*} \mathrm{P}<0.05,{ }^{* *} \mathrm{P}<0.01,{ }^{* * * *} \mathrm{P}<0.001$. Fn, Fusobacterium nucleatum; Fnf, Fn+L-fucose; Con, control.

$\mathrm{P}=0.016 ; \mathrm{SW} 480$ control, $1.000 \pm 0.052 ; F n, 1.400 \pm 0.057$; $\mathrm{P}=0.002)$. However, the protein levels significantly decreased following treatment with fucose (for p-stat3/stat3, HCT116

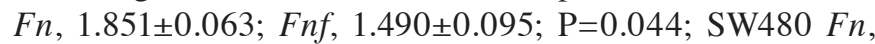
$1.759 \pm 0.075 ;$ Fnf, $1.318 \pm 0.031 ; \mathrm{P}=0.005$. For p-jak2/jak2, HCT116 Fn, 2.272 $\pm 0.098 ;$ Fnf, 1.327 $\pm 0.157 ; \mathrm{P}=0.036$; SW480 $F n, 1.400 \pm 0.057 ;$ Fnf, 1.082 $\pm 0.108 ; \mathrm{P}=0.040)$.

Stat 3 activation is upstream of epithelial-to-mesenchymal transition (EMT) in CRC, which is associated with tumor progression (16). The present study assessed the specific protein markers of EMT, which exist in different types of tumors, such as prostate and colon cancer, and contribute to tumor metastasis (17). As presented in Fig. 5A and B, the expression levels of $\mathrm{N}$-cadherin, $\beta$-catenin and vimentin were higher in HCT116 cells treated with $F n$ compared with the control group (N-cadherin control, $1.00 \pm 0.01 ; F n, 1.34 \pm 0.03$, $\mathrm{P}=0.007 ; \beta$-catenin control, $1.00 \pm 0.02 ; F n, 1.44 \pm 0.03$, $\mathrm{P}=0.006$; vimentin control, $1.00 \pm 0.01 ; F n, 2.19 \pm 0.09$; $\mathrm{P}=0.006$ ). As expected, the expression levels of $\mathrm{N}$-cadherin, $\beta$-catenin and vimentin significantly decreased following treatment with fucose (N-cadherin $F n, 1.34 \pm 0.03 ; F n f, 1.20 \pm 0.01$, $\mathrm{P}=0.048 ; \beta$-catenin Fn, 1.44 $\pm 0.03 ;$ Fnf, 1.26 $\pm 0.03, \mathrm{P}=0.042$; vimentin $F n, 2.19 \pm 0.09 ; F n f, 1.67 \pm 0.07, \mathrm{P}=0.045)$. E-cadherin was expressed at low levels in HCT116 cells treated with Fn 

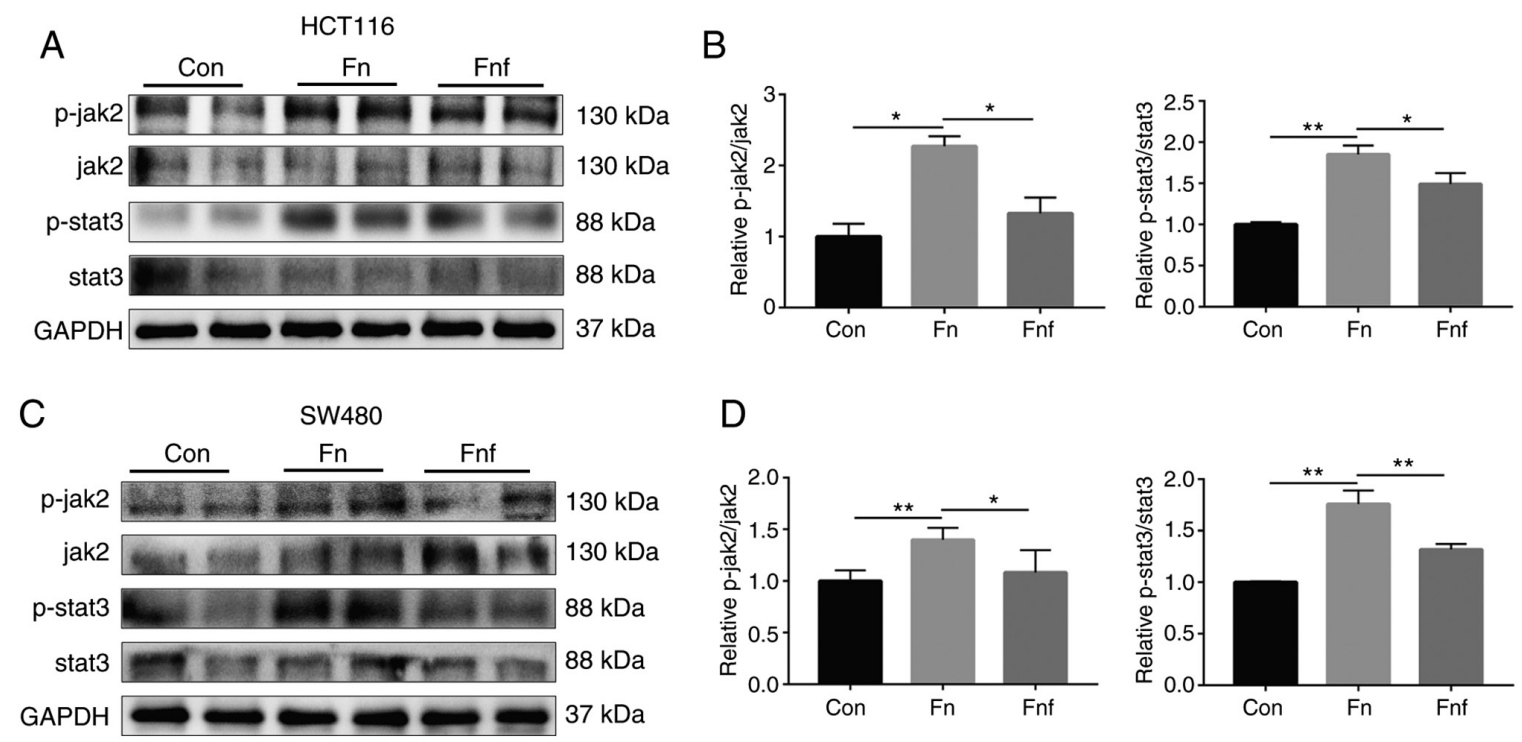

Figure 4. Fucose ameliorate the carcinogenic properties of $F n$ to activate stat3. (A) Representative p-jak2, jak2, p-stat 3 and stat 3 immunoblots of HCT116 cells. Cells were treated with supernatant of $F n$ or $F n f$ for $12 \mathrm{~h}$. GAPDH was used as the loading control. (B) Relative protein levels are presented in histograms. (C) Representative p-jak2, jak2, p-stat3 and stat3 immunoblots of SW480 cells. Cells were treated with supernatant of Fn or Fnf for 12 h. GAPDH was used as the loading control. (D) Relative protein levels are presented in histograms. Data are presented as the mean \pm standard error of the mean of at least three repeated experiments. ${ }^{*} \mathrm{P}<0.05,{ }^{* *} \mathrm{P}<0.01$. p, phosphorylated; Fn, Fusobacterium nucleatum; Fnf, Fn+L-fucose; Con, control.

compared with the other two groups, which demonstrated the change of the EMT pathway (E-cadherin control, 1.00 \pm 0.04 ; Fn, 0.37 $\pm 0.03 ;$ Fnf, $0.72 \pm 0.07 ; \mathrm{P}=0.005$ and $\mathrm{P}=0.040$ ). Similar findings were observed in SW480 cells (Fig. 5C and D).

The stat 3 inhibitor, S3I-201, was used to assess the association between stat 3 activation and EMT following treatment with $F n$. Western blot analysis demonstrated that inhibition of stat 3 activation significantly suppressed the activation of EMT in both HCT116 and SW480 cells (Fig. 5E-H). Taken together, these results suggest that fucose inhibits the carcinogenic properties of $F n$ to activate the stat 3 pathway and EMT.

\section{Discussion}

Recently, several studies have focused on the molecular mechanisms of carcinogenesis of $F n(5,18,19)$; however, methods for resisting the carcinogenic properties remain largely unknown. The present study revealed a distinct role of fucose in ameliorating the carcinogenic properties of $F n$ in vitro.

The results of the present study demonstrated that $F n$ significantly promoted proliferation, migration and invasion of colon cancer cells. Fn was first observed in oral cavity contributing to periodontal diseases (20). Recently, increasing evidence suggests an association between $F n$ and CRC. For example, Mima et al (21) reported that $F n$ was detected in 76/598 (13.0\%) colorectal carcinomas (stages I-IV) and 19/558 (3.4\%) adjacent non-tumor tissues. Furthermore, highly enriched Fusobacterium in CRC tissues is associated with microsatellite instability-high status (22). Fn enrichment may augment myeloid-derived immune cells in CRC, which can inhibit T-cell proliferation and induce apoptosis (23). In addition, $F n$ activates toll-like receptor 4 signaling and downstream PAK1 and NF- $\mathrm{kB}$ in CRC, thus increasing the proliferative and invasive abilities $(18,19)$. Consistent with these findings, the results of the present study demonstrated the carcinogenic properties of $F n$.
Currently, as our research team discovered that fucose is testified to competent in different types of diseases, including colitis, renal ischemia/reperfusion injury and high-fat diet-induced obesity and hepatic steatosis $(10,24,25)$, it has also been demonstrated to impact the microbial ecosystem (11). Notably, fucose may decrease pathogen virulence through certain bacterium's metabolic pathway, such as Salmonella enterica (12). Thus, the present study assessed whether L-fucose can restrain the ability of $F n$ to promote CRC progression. The results demonstrated that $F n$ exhibited less tendency to facilitate the proliferation, migration and invasion of colon cancer cells following treatment with L-fucose. To the best of our knowledge, the present study was the first to demonstrate that L-fucose suppresses flora associated with colon cancer.

Mechanistically, previous studies have demonstrated that $F n$ induces stat 3 expression in macrophages, resulting in M2 polarization and increased tumor-immune cytokine secretion, which alters the tumor microenvironment and promotes colorectal tumor development $(8,26)$. The expression of stat 3 signaling was assessed in the present study. Given that stat3 activation can promote EMT (27), and EMT is an important factor to drive carcinogenesis (16), the change in the expression levels of EMT markers was assessed in the present study. The results demonstrated that the protein expression levels of the EMT markers significantly increased following treatment with $F n$, suggesting that $F n$ may promote EMT in colon cancer cells. In addition, activation of stat 3 signaling and EMT weakened following treatment with L-fucose.

However, there were some limitations in this study. On the one hand, in vivo experiments could further confirm the conclusion and make the study more complete. On the other hand, further studies are needed to explore the mechanism that how fucose impair the carcinogenic properties of $F n$. One hypothesis is that it may alter its metabolism. 
A

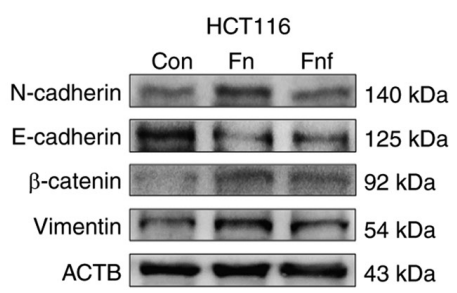

C

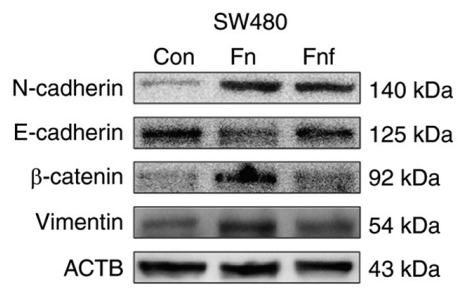

E

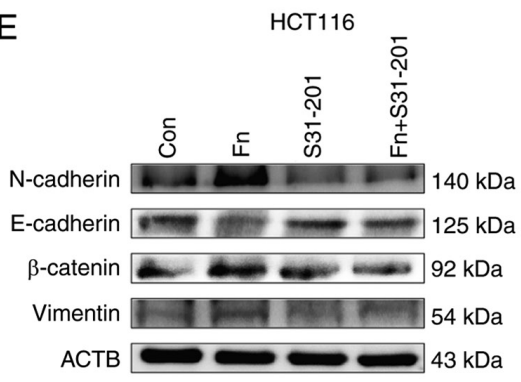

G

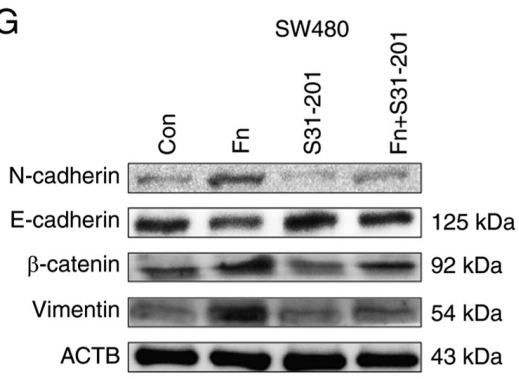

B
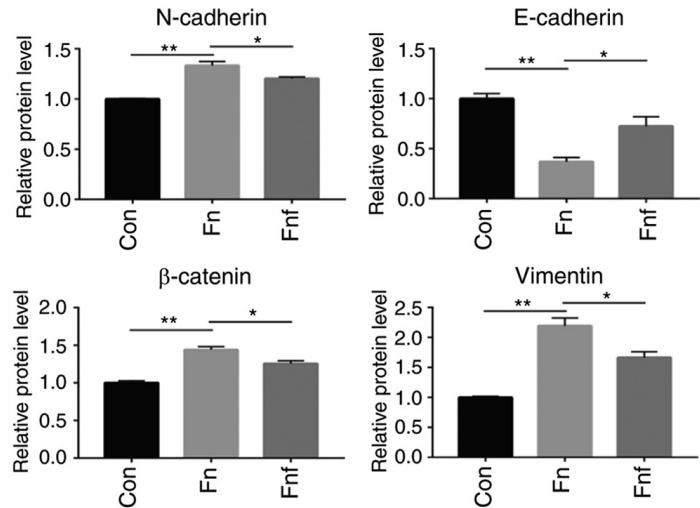

D
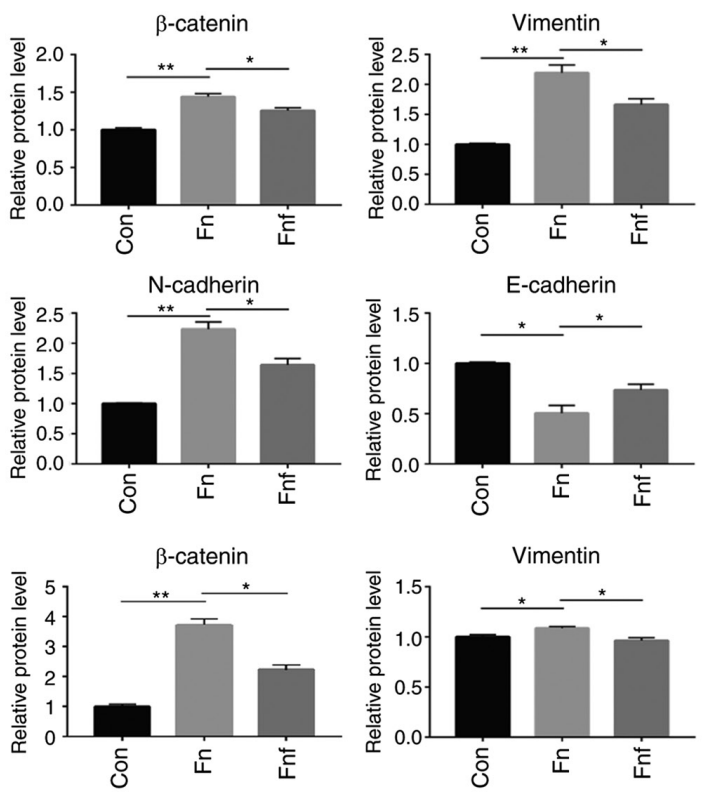

$\mathrm{F}$
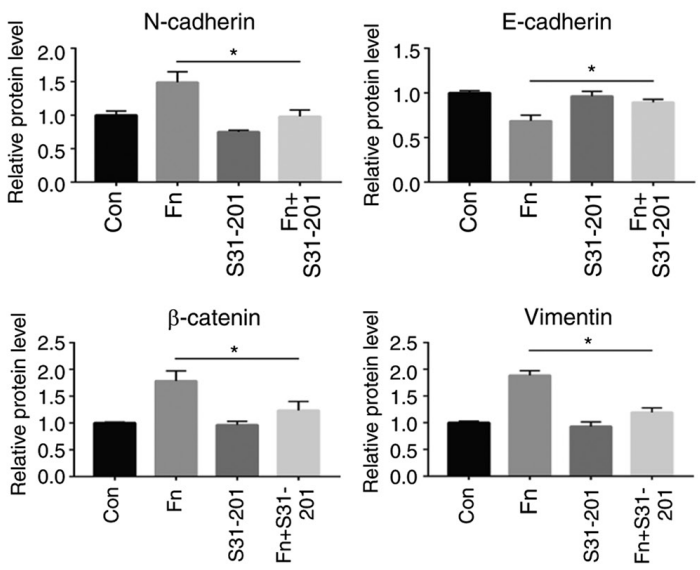

$\mathrm{H}$
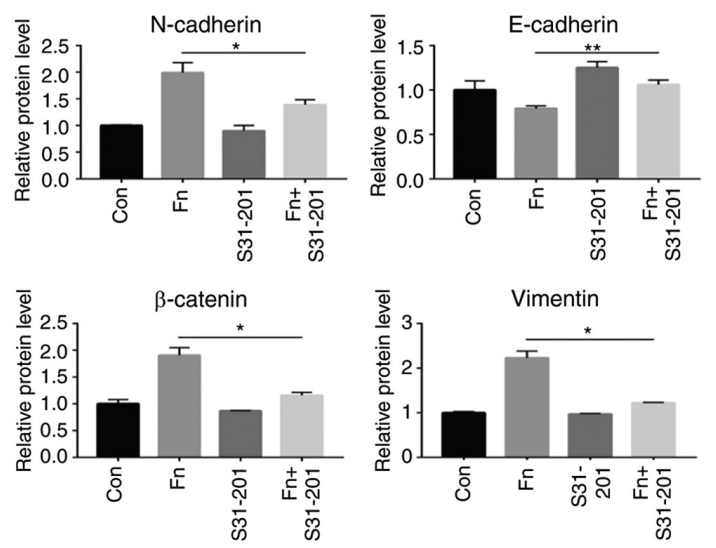

Figure 5. Fucose ameliorates the carcinogenic properties of $F n$ to activate epithelial-to-mesenchymal transition. (A) Representative N-cadherin, E-cadherin, $\beta$-catenin and vimentin immunoblots of HCT116 cells. Cells were treated with supernatant of $F n$ or $F n f$ for 12 h. ACTB was used as the loading control. (B) Relative protein levels are presented in histograms. (C) Representative N-cadherin, E-cadherin, $\beta$-catenin and vimentin immunoblots of SW480 cells. Cells were treated with supernatant of $F n$ or $F n f$ for $12 \mathrm{~h}$. ACTB was used as the loading control. (D) Relative protein levels are presented in histograms. (E) Representative N-cadherin, E-cadherin, $\beta$-catenin and vimentin immunoblots of HCT116 cells. Cells were treated with or without S3I-201 (100 nM) and supernatant of $F n$. (F) Relative protein levels are presented in histograms. (G) Representative N-cadherin, E-cadherin, $\beta$-catenin and vimentin immunoblots of SW480 cells. Cells were treated with or without S3I-201 (100 nM) and supernatant of Fn. (H) Relative protein levels are presented in histogram. Data are presented as the mean \pm standard error of the mean of at least three repeated experiments. ${ }^{*} \mathrm{P}<0.05,{ }^{* *} \mathrm{P}<0.01$. Fn, Fusobacterium nucleatum; Fnf, Fn+L-fucose; Con, control. 
In conclusion, the results of the present study demonstrated that L-fucose ameliorated the carcinogenic properties of $F n$ by suppressing its ability to activate stat 3 and EMT of colon cancer cells in vitro. Thus, L-fucose may serve as a novel therapeutic strategy of microflora-related colon cancer.

\section{Acknowledgements}

Not applicable.

\section{Funding}

The present study was financially supported by the National Natural Science Foundation of China (grant nos. 81800467, 81330014, 81720108006 and 81974062).

\section{Availability of data and materials}

The datasets used and analyzed during the present study are available from the corresponding author upon reasonable request.

\section{Authors' contributions}

CD performed the experiments, analyzed the data and drafted the initial manuscript. XT, WW and WQ helped analyze the data and revised the manuscript for important intellectual content. XF and XD helped culture the bacteria. $\mathrm{XH}$ and $\mathrm{CH}$ designed the present study, provided funding and obtained the grants. All authors have read and approved the final manuscript.

\section{Ethics approval and consent to participate}

Not applicable.

\section{Patient consent for publication}

Not applicable.

\section{Competing interests}

The authors declare that they have no competing interests.

\section{References}

1. Bray F, Ferlay J, Soerjomataram I, Siegel RL, Torre LA and Jemal A: Global cancer statistics 2018: GLOBOCAN estimates of incidence and mortality worldwide for 36 cancers in 185 countries. CA Cancer J Clin 68: 394-424, 2018.

2. O'Keefe SJ: Diet, microorganisms and their metabolites, and colon cancer. Nat Rev Gastroenterol Hepatol 13: 691-706, 2016.

3. Mima K, Nishihara R, Qian ZR, Cao Y, Sukawa Y, Nowak JA, Yang J, Dou R, Masugi Y, Song M, et al: Fusobacterium nucleatum in colorectal carcinoma tissue and patient prognosis. Gut 65: 1973-1980, 2016.

4. Shang FM and Liu HL: Fusobacterium nucleatum and colorectal cancer: A review. World J Gastrointest Oncol 10: 71-81, 2018

5. Chen Y, Chen Y, Zhang J, Cao P, Su W, Deng Y, Zhan N, Fu X, Huang Y and Dong W: Fusobacterium nucleatum promotes metastasis in colorectal cancer by activating autophagy signaling via the upregulation of CARD3 expression. Theranostics 10: 323-339, 2020

6. Brennan CA and Garrett WS: Gut microbiota, inflammation, and colorectal cancer. Annu Rev Microbiol 70: 395-411, 2016.
7. Abed J,Emgård JE,Zamir G, Faroja M, Almogy G, Grenov A, Sol A Naor R, Pikarsky E, Atlan KA, et al: Fap2 mediates fusobacterium nucleatum colorectal adenocarcinoma enrichment by binding to tumor-expressed Gal-GalNAc. Cell Host Microbe 20: 215-225, 2016.

8. Yu YN, Yu TC, Zhao HJ, Sun TT, Chen HM, Chen HY, An HF, Weng YR, Yu J, Li M, et al: Berberine may rescue fusobacterium nucleatum-induced colorectal tumorigenesis by modulating the tumor microenvironment. Oncotarget 6: 32013-32026, 2015.

9. Goto Y, Uematsu S and Kiyono H: Epithelial glycosylation in gut homeostasis and inflammation. Nat Immunol 17: 1244-1251, 2016.

10. He R, Li Y, Han C, Lin R, Qian W and Hou X: L-Fucose ameliorates DSS-induced acute colitis via inhibiting macrophage M1 polarization and inhibiting NLRP3 inflammasome and NF- $\kappa B$ activation. Int Immunopharmacol 73: 379-388, 2019.

11. Ke J, Li Y, Han C, He R, Lin R, Qian W and Hou X: Fucose ameliorate intestinal inflammation through modulating the crosstalk between bile acids and gut microbiota in a chronic colitis murine model. Inflamm Bowel Dis 26: 863-873, 2020.

12. Pickard JM, Maurice CF, Kinnebrew MA, Abt MC, Schenten D, Golovkina TV, Bogatyrev SR, Ismagilov RF, Pamer EG, Turnbaugh PJ and Chervonsky AV: Rapid fucosylation of intestinal epithelium sustains host-commensal symbiosis in sickness. Nature 514: 638-641, 2014.

13. Neish AS: Microbes in gastrointestinal health and disease. Gastroenterology 136: 65-80, 2009.

14. Kyoung AK, Ryu YS, Piao MJ, Shilnikova K, Kang HK, Yi JM, Boulanger M, Paolillo R, Bossis G, Yoon SY, et al: DUOX2-mediated production of reactive oxygen species induces epithelial mesenchymal transition in 5-fluorouracil resistant human colon cancer cells. Redox Biol 17: 224-235, 2018.

15. Yu H, Lee H, Herrmann A, Buettner R and Jove R: Revisiting STAT3 signalling in cancer: New and unexpected biological functions. Nat Rev Cancer 14: 736-746, 2014.

16. Fan Y,Mao R and Yang J: NF-kB and STAT3 signaling pathways collaboratively link inflammation to cancer. Protein Cell 4: 176-185, 2013.

17. Chen T, You Y, Jiang H and Wang ZZ: Epithelial-mesenchymal transition (EMT): A biological process in the development, stem cell differentiation, and tumorigenesis. J Cell Physiol 232: 3261-3272, 2017.

18. Chen Y, Peng Y, Yu J, Chen T, Wu Y, Shi L, Li Q, Wu J and $\mathrm{Fu} \mathrm{X}$ : Invasive fusobacterium nucleatum activates beta-catenin signaling in colorectal cancer via a TLR4/P-PAK1 cascade. Oncotarget 8: 31802-31814, 2017.

19. Yang Y, Weng W, Peng J, Hong L, Yang L, Toiyama Y, Gao R, Liu M, Yin M, Pan C, et al: Fusobacterium nucleatum increases proliferation of colorectal cancer cells and tumor development in mice by activating toll-like receptor 4 signaling to nuclear factor- $\kappa \mathrm{B}$, and up-regulating expression of MicroRNA-21. Gastroenterology 152: 851-866.e824, 2017.

20. Brennan CA and Garrett WS: Fusobacterium nucleatum-symbiont, opportunist and oncobacterium. Nat Rev Microbiol 17: 156-166, 2019.

21. Mima K, Sukawa Y, Nishihara R, Qian ZR, Yamauchi M, Inamura K, Kim SA, Masuda A, Nowak JA, Nosho K, et al: Fusobacterium nucleatum and T cells in colorectal carcinoma. JAMA Oncol 1: 653-661, 2015.

22. Nosho K, Sukawa Y, Adachi Y, Ito M, Mitsuhashi K, Kurihara H, Kanno S, Yamamoto I, Ishigami K, Igarashi H, et al: Association of fusobacterium nucleatum with immunity and molecular alterations in colorectal cancer. World J Gastroenterol 22: 557-566, 2016.

23. Kostic AD, Chun E, Robertson L, Glickman JN, GalliniCA, MichaudM, Clancy TE, Chung DC, Lochhead P, Hold GL, et al: Fusobacterium nucleatum potentiates intestinal tumorigenesis and modulates the tumor-immune microenvironment. Cell Host Microbe 14: 207-215, 2013.

24. Howard MC, Nauser CL, Farrar CA, Wallis R and Sacks SH: 1-Fucose prevention of renal ischaemia/reperfusion injury in Mice. FASEB J 34: 822-834, 2020.

25. Wu G, Niu M, Tang W, Hu J, Wei G, He Z, Chen Y, Jiang Y and Chen P: L-Fucose ameliorates high-fat diet-induced obesity and hepatic steatosis in mice. J Transl Med 16: 344, 2018.

26. Chen T, Li Q, Wu J, Wu Y, Peng W, Li H, Wang J, Tang X, Peng Y and $\mathrm{Fu} \mathrm{X}$ : Fusobacterium nucleatum promotes M2 polarization of macrophages in the microenvironment of colorectal tumours via a TLR4-dependent mechanism. Cancer Immunol Immunother 67: 1635-1646, 2018.

27. RokavecM,ÖnerMG,LiH,JackstadtR,JiangL,Lodygin D,KallerM, Horst D, Ziegler PK, Schwitalla S, et al: IL-6R/STAT3/miR-34a feedback loop promotes EMT-mediated colorectal cancer invasion and metastasis. J Clin Invest 124: 1853-1867, 2014.

This work is licensed under a Creative Commons Attribution-NonCommercial-NoDerivatives 4.0 International (CC BY-NC-ND 4.0) License. 